\title{
SEMIORTHOGONAL SPLINE WAVELETS APPROXIMATION FOR FREDHOLM INTEGRO-DIFFERENTIAL EQUATIONS
}

\author{
M. LAKESTANI, M. RAZZAGHI, AND M. DEHGHAN
}

Received 18 February 2005; Revised 11 May 2005; Accepted 20 June 2005

A method for solving the nonlinear second-order Fredholm integro-differential equations is presented. The approach is based on a compactly supported linear semiorthogonal $B$-spline wavelets. The operational matrices of derivative for $B$-spline scaling functions and wavelets are presented and utilized to reduce the solution of Fredholm integrodifferential to the solution of algebraic equations. Illustrative examples are included to demonstrate the validity and applicability of the technique.

Copyright (c) 2006 M. Lakestani et al . This is an open access article distributed under the Creative Commons Attribution License, which permits unrestricted use, distribution, and reproduction in any medium, provided the original work is properly cited.

\section{Introduction}

Integro-differential equations have gained a lot of interest in many application fields, such as biological, physical, and engineering problems. Therefore, their numerical treatment is desired [2]. While several numerical methods for approximating the solution of Volterra integro-differential equations are known for Fredholm integro-differential equations, not many are discussed in the literature [8].

Consider the linear second-order Fredholm integro-differential of the form

$$
\sum_{i=0}^{2} \mu_{i}(x) y^{(i)}(x)=g(x)+\int_{0}^{1} K(x, t) y(t) d t, \quad 0 \leq x \leq 1,
$$

and the nonlinear second-order Fredholm integro-differential of the form

$$
\sum_{i=0}^{2} \mu_{i}(x) y^{(i)}(x)=f\left(x, y(x), \int_{0}^{1} K(x, t, y(t)) d t\right), \quad 0 \leq x \leq 1,
$$

with

$$
y(0)=y_{0}, \quad y(1)=y_{1},
$$

Hindawi Publishing Corporation

Mathematical Problems in Engineering

Volume 2006, Article ID 96184, Pages 1-12

DOI 10.1155/MPE/2006/96184 
where $\mu_{i}, i=0,1,2, g, f$, and $K$, are given functions in $L^{2}[0,1], y_{0}$ and $y_{1}$ are given real numbers and $y$ is the unknown function to be found.

For linear first-order Fredholm integro-differential of the form (1.1), Linz [7] considered numerical methods by transforming it into a second kind of integral equation, and, in [12], Volk applied projection methods. For nonlinear first-order of the form (1.2), an iterative procedure was developed in Phillips [10], direct numerical spline methods were discussed in [8], and a collocation procedure with spline functions was introduced in [2]. For linear second-order Fredholm integro-differential of the form (1.1), wavelet methods, by using the Daubechies orthonormal scaling functions, were presented in [3].

In recent years, the application of methods based on wavelets have influenced many areas of applied mathematics. In areas such as the numerical analysis of differential equations, wavelets are recognized as a powerful tool. Another area in which the wavelet is gaining considerable attention is the study of integral equations. Wavelets can be separated into two distinct types; orthogonal and semiorthogonal [4]. Publications on integral equation methods have shown a marked preference for orthogonal wavelets [9]. This is probably because the original wavelets, which were widely used for signal processing, were primarily orthogonal. In signal processing applications, unlike integral equation methods, the wavelet itself is never constructed since only its scaling function and coefficients are needed. However, orthogonal wavelets either have infinite support or a nonsymmetric and in some cases, fractal nature. These properties can make them a poor choice for characterization of a function. In contrast, the semiorthogonal wavelets have finite support, both even and odd symmetry and simple analytical expressions, ideal attributes of a basis function [9].

In the present paper, we apply compactly supported linear semiorthogonal (SO) $B$ spline wavelets, specially constructed for the bounded interval to solve the nonlinear second-order Fredholm integro-differential equation of the form (1.2). The use of SO compactly supported spline wavelets is justified by their interesting properties $[1,6]$.

Our method consists of reducing (1.2) and (1.3) to a set of algebraic equations by expanding unknown function as linear $B$-spline wavelets with unknown coefficients. The properties of linear $B$-spline scaling functions and wavelets are then utilized to evaluate the unknown coefficients.

The paper is organized as follows. In Section 2, we describe the formulation of the $B$ spline scaling functions and wavelets on $[0,1]$, and derive the operational matrices of derivative required for our subsequent development. In Section 3, the proposed method is used to approximate the solution of nonlinear second-order Fredholm integrodifferential equation. In Section 4, we report our numerical finding and demonstrate the accuracy of the proposed numerical scheme by considering numerical examples.

\section{2. $B$-spline scaling functions and wavelets on $[0,1]$}

When semiorthogonal wavelets are constructed from $B$-splines of order $m$, the lowest octave level $j=j_{0}$ is determined in [5] by

$$
2^{j_{0}} \geq 2 m-1,
$$

so as to give a minimum of one complete wavelet on the interval $[0,1]$. In this paper, we 
will use a wavelet generated by a linear spline $(m=2)$ cardinal $B$-spline function. From (2.1), the second-order $B$-spline lowest level, which must be an integer, is determined to be $j_{0}=2$. This constrains all octave levels to $j \geq 2$.

As in the case with all semiorthogonal wavelets, the second-order $B$-splines also serve as scaling functions. The second-order $B$-splines/scaling functions are given by

$$
\phi_{j, k}(x)= \begin{cases}x_{j}-k, & k \leq x_{j}<k+1, \\ 2-\left(x_{j}-k\right), & k+1 \leq x_{j}<k+2, k=0, \ldots, 2^{j}-2, \\ 0, & \text { otherwise, }\end{cases}
$$

with the respective left- and right-hand side boundary scaling functions

$$
\begin{aligned}
\phi_{j, k}(x) & = \begin{cases}2-\left(x_{j}-k\right), & 0 \leq x_{j}<1, k=-1, \\
0, & \text { otherwise, }\end{cases} \\
\phi_{j, k}(x) & = \begin{cases}x_{j}-k, & k \leq x_{j}<k+1, k=2^{j}-1, \\
0, & \text { otherwise. }\end{cases}
\end{aligned}
$$

The actual coordinate position $x$ is related to $x_{j}$, according to $x_{j}=2^{j} x$. The second-order $B$-spline wavelets are given by

$$
\psi_{j, k}(x)=\frac{1}{6} \begin{cases}x_{j}-k, & k \leq x_{j}<k+1 / 2, \\ 4-7\left(x_{j}-k\right), & k+1 / 2 \leq x_{j}<k+1, \\ -19+16\left(x_{j}-k\right), & k+1 \leq x_{j}<k+3 / 2, \\ 29-16\left(x_{j}-k\right), & k+3 / 2 \leq x_{j}<k+2, k=0, \ldots, 2^{j}-3, \\ -17+7\left(x_{j}-k\right), & k+2 \leq x_{j}<k+5 / 2, \\ 3-\left(x_{j}-k\right), & k+5 / 2 \leq x_{j}<k+3, \\ 0, & \text { otherwise, }\end{cases}
$$

with the respective left- and right-hand side boundary wavelets

$$
\begin{aligned}
& \psi_{j, k}(x)=\frac{1}{6} \begin{cases}-6+23 x_{j}, & 0 \leq x_{j}<1 / 2, \\
14-17 x_{j}, & 1 / 2 \leq x_{j}<1, \\
-10+7 x_{j}, & 1 \leq x_{j}<3 / 2, k=-1, \\
2-x_{j}, & 3 / 2 \leq x_{j}<2, \\
0, & \text { otherwise, }\end{cases} \\
& \psi_{j, k}(x)=\frac{1}{6} \begin{cases}2-\left(k+2-x_{j}\right), & k \leq x_{j}<k+1 / 2, \\
-10+7\left(k+2-x_{j}\right), & k+1 / 2 \leq x_{j}<k+1, \\
14-17\left(k+2-x_{j}\right), & k+1 \leq x_{j}<k+3 / 2, k=2^{j}-2 \\
-6+23\left(k+2-x_{j}\right), & k+3 / 2 \leq x_{j}<k+2, \\
0, & \text { otherwise. }\end{cases}
\end{aligned}
$$


4 Semiorthogonal spline wavelets

From (2.2)-(2.7), we get

$$
\begin{aligned}
\phi_{i, j}(x)= & \frac{1}{2} \phi_{i+1,2 j}(x)+\phi_{i+1,2 j+1}(x)+\frac{1}{2} \phi_{i+1,2 j+2}(x), \quad i=2,3, \ldots, j=0,1,2, \ldots, 2^{i}-2, \\
\phi_{i,-1}(x)= & \phi_{i+1,-1}(x)+\frac{1}{2} \phi_{i+1,0}(x), \quad i=2,3, \ldots, \\
\phi_{i, 2^{i}-1}(x)= & \phi_{i+1,2^{i+1}-1}+\frac{1}{2} \phi_{i+1,2^{i+1}-2}(x), \quad i=2,3, \ldots, \\
& -\frac{1}{2} \phi_{i+1,2 j+3}(x)+\frac{1}{12} \phi_{i+1,2 j+4}(x), \quad i=2,3, \ldots, j=0,1,2, \ldots, 2^{i}-3, \\
\psi_{i, j}(x)= & \frac{1}{12} \phi_{i+1,2 j}(x)-\frac{1}{2} \phi_{i+1,2 j+1}(x)+\frac{5}{6} \phi_{i+1,2 j+2}(x) \\
\psi_{i,-1}(x)= & -\phi_{i+1,-1}(x)+\frac{11}{12} \phi_{i+1,0}(x)-\frac{1}{2} \phi_{i+1,1}(x)+\frac{1}{12} \phi_{i+1,2}(x), \quad i=2,3, \ldots \\
\psi_{i, 2^{i}-2}(x)= & -\phi_{i+1,2^{i+1}-2}(x)+\frac{11}{12} \phi_{i+1,2^{i+1}-3}(x)-\frac{1}{2} \phi_{i+1,2^{i+1}-4}(x)+\frac{1}{12} \phi_{i+1,2^{i+1}-5}, \quad i=2,3, \ldots
\end{aligned}
$$

2.1. Function approximation. For any fixed positive integer $M$, a function $f(x)$ defined over $[0,1]$ may be represented by $B$-spline scaling functions as

$$
f(x)=\sum_{k=-1}^{2^{M+1}-1} s_{k} \phi_{M+1, k}=S^{T} \Phi_{M+1}
$$

where

$$
\begin{gathered}
S=\left[s_{-1}, s_{0}, \ldots, s_{2^{M+1}-1}\right]^{T}, \\
\Phi_{M+1}=\left[\phi_{M+1,-1}, \phi_{M+1,0}, \ldots, \phi_{M+1,2^{M+1}-1}\right]^{T},
\end{gathered}
$$

with

$$
s_{k}=\int_{0}^{1} f(x) \tilde{\phi}_{M+1, k}(x) d x, \quad k=-1,0, \ldots, 2^{M+1}-1,
$$

where $\tilde{\phi}_{M+1, k}(x)$ are dual functions of $\phi_{M+1, k}$. These can be obtained by linear combinations of $\phi_{M+1, k}, k=-1, \ldots, 2^{M+1}-1$, as follows. Let $\widetilde{\Phi}_{M+1}$ be the dual functions of $\Phi_{M+1}$ given by

$$
\tilde{\Phi}_{M+1}=\left[\tilde{\phi}_{M+1,-1}, \tilde{\phi}_{M+1,0}, \ldots, \tilde{\phi}_{M+1,2^{M+1}-1}\right]^{T} .
$$

Using (2.11) and (2.13), we get

$$
\int_{0}^{1} \widetilde{\Phi}_{M+1} \Phi_{M+1}^{T} d x=I_{1}
$$


where $I_{1}$ is $\left(2^{(M+1)}+1\right) \times\left(2^{(M+1)}+1\right)$ identity matrix. Let

$$
P_{M+1}=\int_{0}^{1} \Phi_{M+1} \Phi_{M+1}^{T} d x
$$

The entry $\left(P_{M+1}\right)_{i, j}$ of the matrix $P_{M+1}$ in (2.15) is calculated from

$$
\int_{0}^{1} \phi_{M+1, i}(x) \phi_{M+1, j}(x) d x
$$

Using (2.2)-(2.4), (2.11) and (2.16), we get a symmetric $\left(2^{M+1}+1\right) \times\left(2^{M+1}+1\right)$ matrix for $P_{M+1}$, which is given by

$$
P_{M+1}=\frac{1}{2^{M-1}}\left[\begin{array}{ccccc}
\frac{1}{12} & \frac{1}{24} & 0 & \cdots & 0 \\
\frac{1}{24} & \frac{1}{6} & \frac{1}{24} & \cdots & 0 \\
\vdots & \ddots & \ddots & \ddots & \vdots \\
0 & \cdots & \frac{1}{24} & \frac{1}{6} & \frac{1}{24} \\
0 & \cdots & 0 & \frac{1}{24} & \frac{1}{12}
\end{array}\right] .
$$

From (2.14) and (2.15), we get

$$
\tilde{\Phi}_{M+1}=\left(P_{M+1}\right)^{-1} \Phi_{M+1}
$$

Furthermore, a function $f(x)$ defined over $[0,1]$ may be represented by $B$-spline wavelets as

$$
f(x)=\sum_{k=-1}^{3} c_{k} \phi_{2, k}(x)+\sum_{i=2}^{\infty} \sum_{j=-1}^{2^{i}-2} d_{i, j} \psi_{i, j}(x)
$$

If the infinite series in (2.19) is truncated at $M$, then (2.19) can be written as

$$
f(x) \simeq \sum_{k=-1}^{3} c_{k} \phi_{2, k}(x)+\sum_{i=2}^{M} \sum_{j=-1}^{2^{i}-2} d_{i, j} \psi_{i, j}(x)=\alpha^{T} \Psi(x)
$$

where $\phi_{2, k}$ and $\psi_{i, j}$ are scaling and wavelets functions, respectively, and $\alpha$ and $\Psi$ are 
6 Semiorthogonal spline wavelets

$\left(2^{(M+1)}+1\right) \times 1$ vectors given by

$$
\begin{aligned}
\alpha & =\left[c_{-1}, c_{0}, \ldots, c_{3}, d_{2,-1}, \ldots, d_{2,2}, d_{3,-1}, \ldots, d_{3,6}, \ldots, d_{M,-1}, \ldots, d_{M, 2^{M}-2}\right]^{T}, \\
\Psi & =\left[\phi_{2,-1}, \phi_{2,0}, \ldots, \phi_{2,3}, \psi_{2,-1}, \ldots, \psi_{2,2}, \psi_{3,-1}, \ldots, \psi_{3,6}, \ldots, \psi_{M,-1}, \ldots, \psi_{M, 2^{M}-2}\right]^{T} .
\end{aligned}
$$

2.2. The operational matrices of derivative. The differentiation of the vectors $\Phi_{M+1}$ and $\Psi$ in (2.11) and (2.22) can be expressed as

$$
\Phi_{M+1}^{\prime}=D_{\Phi} \Phi_{M+1}, \quad \Psi^{\prime}=D_{\Psi} \Psi
$$

where $D_{\Phi}$ and $D_{\Psi}$ are $\left(2^{M+1}+1\right) \times\left(2^{M+1}+1\right)$ operational matrices of derivative for $B$ spline scaling functions and wavelets, respectively. Using (2.10), (2.12), and (2.18) the matrix $D_{\Phi}$ can be obtained as

$$
D_{\Phi}=\int_{0}^{1} \Phi_{M+1}^{\prime}(t) \tilde{\Phi}_{M+1}^{T}(t) d t=\left(\int_{0}^{1} \Phi_{M+1}^{\prime}(t) \Phi_{M+1}^{T}(t) d t\right)\left(P_{M+1}\right)^{-1}=E\left(P_{M+1}\right)^{-1}
$$

where

$$
E=\int_{0}^{1} \Phi_{M+1}^{\prime}(t) \Phi_{M+1}^{T}(t) d t
$$

In (2.25), $E$ is $\left(2^{M+1}+1\right) \times\left(2^{M+1}+1\right)$ matrix given by

$$
E=\left[\begin{array}{ccc}
\int_{0}^{1} \phi_{M+1,-1}^{\prime}(t) \phi_{M+1,-1}(t) d t & \ldots & \int_{0}^{1} \phi_{M+1,-1}^{\prime}(t) \phi_{M+1,2^{M+1}-1}(t) d t \\
\vdots & \ddots & \vdots \\
\int_{0}^{1} \phi_{M+1,2^{M+1}-1}^{\prime}(t) \phi_{M+1,-1}(t) d t & \ldots & \int_{0}^{1} \phi_{M+1,2^{M+1}-1}^{\prime}(t) \phi_{M+1,2^{M+1}-1}(t) d t
\end{array}\right] .
$$

Since the element $\phi_{M+1, k}$ in the vector $\Phi_{M+1}$ given in $(2.11)$ is nonzero between $k / 2^{M+1}$ and $(k+2) / 2^{M+1}$, for any entries of $E_{j, k}$, we have

$$
\begin{aligned}
E_{j, k} & =\int_{0}^{1} \phi_{M+1, j}^{\prime}(t) \phi_{M+1, k}(t) d t=\int_{k / 2^{M+1}}^{(k+2) / 2^{M+1}} \phi_{M+1, j}^{\prime}(t) \phi_{M+1, k}(t) d t \\
& =\int_{k / 2^{M+1}}^{(k+1) / 2^{M+1}} \phi_{M+1, j}^{\prime}(t) \phi_{M+1, k}(t) d t+\int_{(k+1) / 2^{M+1}}^{(k+2) / 2^{M+1}} \phi_{M+1, j}^{\prime}(t) \phi_{M+1, k}(t) d t \\
& =\int_{k / 2^{M+1}}^{(k+1) / 2^{M+1}} 2^{M+1} \phi_{M+1, k}(t) d t+\int_{(k+1) / 2^{M+1}}^{(k+2) / 2^{M+1}}-2^{M+1} \phi_{M+1, k}(t) d t .
\end{aligned}
$$


From (2.27), we get

$$
E=\left[\begin{array}{ccccc}
-\frac{1}{2} & -\frac{1}{2} & & & \\
\frac{1}{2} & 0 & -\frac{1}{2} & & \\
& \ddots & \ddots & \ddots & \\
& & \frac{1}{2} & 0 & -\frac{1}{2} \\
& & & \frac{1}{2} & \frac{1}{2}
\end{array}\right]
$$

The matrix $D_{\Psi}$ can be obtained by considering

$$
\Psi=G \Phi_{M+1},
$$

where $G$ is a $\left(2^{M+1}+1\right) \times\left(2^{M+1}+1\right)$ matrix, which can be calculated as follows. Let

$$
\begin{aligned}
& \Phi_{j}=\left[\phi_{j,-1}, \phi_{j, 0}, \ldots, \phi_{j, 2^{j}-1}\right]^{T}, \\
& \Psi_{j}=\left[\psi_{j,-1}, \psi_{j, 0}, \ldots, \psi_{j, 2^{j}-2}\right]^{T} .
\end{aligned}
$$

Using (2.8) and (2.30), we get

$$
\Phi_{j}=\beta_{j} \Phi_{j+1}
$$

with

$$
\beta_{j}=\left[\begin{array}{ccccccccc}
1 & \frac{1}{2} & & & & & & \\
& \frac{1}{2} & 1 & \frac{1}{2} & & & & \\
& & \frac{1}{2} & 1 & \frac{1}{2} & & \\
& & & \ddots & \ddots & \ddots & & \\
& & & & \frac{1}{2} & 1 & \frac{1}{2} & \\
& & & & & & & \frac{1}{2} & 1
\end{array}\right],
$$

where $\beta_{j}, j=2,3, \ldots$, is $\left(2^{j}+1\right) \times\left(2^{j+1}+1\right)$ matrix. From (2.9) and (2.31), we have

$$
\Psi_{j}=L_{j} \Phi_{j+1}
$$


8 Semiorthogonal spline wavelets

with

$L_{j}$

$$
\begin{aligned}
& {\left[\begin{array}{llll}
-1 & \frac{11}{12} & -\frac{1}{2} & \frac{1}{12}
\end{array}\right.} \\
& \frac{1}{12} \quad-\frac{1}{2} \quad \frac{5}{6} \quad-\frac{1}{2} \quad \frac{1}{12} \\
& \frac{1}{12} \quad-\frac{1}{2} \quad \frac{5}{6} \quad-\frac{1}{2} \quad \frac{1}{12} \\
& \frac{1}{12} \quad-\frac{1}{2} \quad \frac{5}{6} \quad-\frac{1}{2} \quad \frac{1}{12} \\
& = \\
& \ddots \quad \ddots \quad \ddots \quad \ddots \quad \ddots . \\
& \frac{1}{12} \quad-\frac{1}{2} \quad \frac{5}{6} \quad-\frac{1}{2} \quad \frac{1}{12} \\
& \frac{1}{12} \quad-\frac{1}{2} \quad \frac{5}{6} \quad-\frac{1}{2} \quad \frac{1}{12} \\
& \frac{1}{12} \quad-\frac{1}{2} \quad \frac{11}{12} \quad-1
\end{aligned}
$$

where $L_{j}, j=2,3, \ldots$, is $2^{j} \times\left(2^{j+1}+1\right)$ matrix.

From (2.32) and (2.34), we get

$$
\begin{aligned}
& \Phi_{j}=\beta_{j} \times \beta_{j+1} \times \cdots \times \beta_{M} \Phi_{M+1}, \\
& \Psi_{j}=L_{j} \times \beta_{j+1} \times \cdots \times \beta_{M} \Phi_{M+1} .
\end{aligned}
$$

Using (2.22) and (2.36), we have

$$
G=\left[\begin{array}{c}
\beta_{2} \times \beta_{3} \times \cdots \times \beta_{M} \\
-------- \\
L_{2} \times \beta_{3} \times \cdots \times \beta_{M} \\
-------- \\
\vdots \\
L_{M-2} \times \beta_{M-1} \times \beta_{M} \\
-------- \\
L_{M-1} \beta_{M} \\
------- \\
L_{M}
\end{array}\right] .
$$

From (2.23), (2.24), and (2.37), we get

$$
\Psi^{\prime}=G \Phi_{M+1}^{\prime}=G D_{\Phi} \Phi_{M+1}=G E\left(P_{M+1}\right)^{(-1)} \Phi_{M+1}=G E\left(P_{M+1}\right)^{(-1)} G^{-1} \Psi=D_{\Psi} \Psi
$$


where

$$
D_{\Psi}=G E\left(P_{M+1}\right)^{-1} G^{-1} .
$$

\section{Nonlinear second-order Fredholm integro-differential equations}

In this section, we solve nonlinear second-order Fredholm integro-differential equations of the form in (1.2) with (1.3) by using $B$-spline wavelets. For this purpose, we first write (1.2) as

$$
\sum_{i=0}^{2} \mu_{i}(x) y^{(i)}(x)=f(x, y(x), z(x)), \quad 0 \leq x \leq 1
$$

where

$$
z(x)=\int_{0}^{1} K(x, t, y(t)) d t
$$

We now use (2.19) to approximate $y(x)$ and $z(x)$ as

$$
\begin{gathered}
y(x)=C^{T} \Psi(x), \\
z(x)=\int_{0}^{1} K\left(x, t, C^{T} \Psi(t)\right) d t,
\end{gathered}
$$

where $\Psi(x)$ is defined in (2.22), and $C$ is $\left(2^{(M+1)}+1\right) \times 1$ unknown vector defined similarly to $\alpha$ in (2.21). We can approximate (3.4), using Newton-Cotes integration techniques $[11]$ as

$$
z(x)=\int_{0}^{1} K\left(x, t, C^{T} \Psi(t)\right)=\sum_{i=1}^{n} \omega_{i} K\left(x, t_{i}, C^{T} \Psi\left(t_{i}\right)\right)=F(x, C),
$$

where $\omega_{i}$ and $t_{i}$ are weight and nodes of Newton-Cotes integration techniques. Using (2.38) and (3.3), we get

$$
\begin{gathered}
y^{\prime}(x)=C^{T} \Psi^{\prime}(x)=C^{T} D_{\Psi} \Psi(x), \\
y^{\prime \prime}(x)=C^{T} D_{\Psi}^{2} \Psi(x) .
\end{gathered}
$$

From (3.1), (3.5), and (3.6), we get

$$
\mu_{0}(x) C^{T} \Psi(x)+\mu_{1}(x) C^{T} D_{w} \Psi(x)+\mu_{2}(x) C^{T} D_{w}^{2} \Psi(x)=f\left(x, C^{T} \Psi(x), F(x, C)\right) .
$$

Also, using (1.3) and (3.3), we have

$$
C^{T} \Psi(0)=y_{0}, \quad C^{T} \Psi(1)=y_{1} .
$$

To find the solution $y(x)$ in (3.1), we first collocate (3.7) in $x_{i}=(2 i-1) /\left(2^{M+2}-2\right)$, $i=1,2, \ldots, 2^{M+1}-1$, the resulting equation generates $2^{M+1}-1$ nonlinear equations which can be solved using Newton's iterative method. The initial values required to start Newton's method have been chosen by taking $y(x)$ as linear function between $y(0)=y_{0}$ and 
Table 4.1. Absolute relative error for Example 4.1.

\begin{tabular}{ccc}
\hline$x_{i}$ & $M=4$ & $M=6$ \\
\hline 0.0 & 0.0 & 0.0 \\
0.1 & $5.0 \times 10^{-5}$ & $3.1 \times 10^{-6}$ \\
0.2 & $4.4 \times 10^{-5}$ & $2.7 \times 10^{-6}$ \\
0.3 & $4.0 \times 10^{-5}$ & $2.5 \times 10^{-6}$ \\
0.4 & $3.9 \times 10^{-5}$ & $2.5 \times 10^{-6}$ \\
0.5 & $8.4 \times 10^{-5}$ & $5.3 \times 10^{-7}$ \\
0.6 & $3.4 \times 10^{-5}$ & $2.1 \times 10^{-6}$ \\
0.7 & $2.8 \times 10^{-5}$ & $1.7 \times 10^{-6}$ \\
0.8 & $2.4 \times 10^{-5}$ & $1.5 \times 10^{-6}$ \\
0.9 & $2.2 \times 10^{-5}$ & $1.4 \times 10^{-6}$ \\
1.0 & 0.0 & 0.0 \\
\hline
\end{tabular}

$y(1)=y_{1}$. The total unknowns for vector $C$ in (3.3) is $2^{M+1}+1$. These can be obtained by using (3.7) and (3.8).

\section{Numerical examples}

We applied the method presented in this paper and solved three examples given in $[2,3]$. This method differs from the collocation procedure with spline functions presented in [2] and Daubechies orthonormal scaling functions given in [3], and thus these could be used as a basis for comparison. Example 4.1 was carried out in [2] by using spline functions with $m=1$ and 2, where $m$ equals to the order of spline functions. Examples 4.2 and 4.3 were carried out in [3] in both the wavelet Galerkin and the wavelet collocation methods by using Daubechies 6, that is, $M=6$.

Example 4.1. Consider first-order Fredholm integro-differential equation [2]

$$
y^{\prime}(x)=-y(x)+\int_{0}^{1} y(t) d t+e^{(-1)}-1, \quad 0 \leq x \leq 1, \quad y(0)=1 .
$$

The exact solution of this problem is $e^{-x}$. We applied the method presented in Section 3 and solved (4.1). The absolute relative error for $M=4$ and $M=6$ are shown in Table 4.1. The absolute relative error in [2] with step size $h=0.1$ at the point 0.4 are given as $1.5 \times$ $10^{-2}$ and $1.8 \times 10^{-3}$, for $m=1$ and $m=2$, respectively.

Example 4.2. Consider second-order Fredholm integro-differential equation [3]

$$
y^{\prime \prime}(x)+4 x y^{\prime}=-\frac{8 x^{4}}{\left(x^{2}+1\right)^{3}}-2 \int_{0}^{1} \frac{t^{2}+1}{\left(x^{2}+1\right)^{2}} y(t) d t, \quad y(0)=1, \quad y(1)=\frac{1}{2} .
$$

Exact solution of this problem is $\left(x^{2}+1\right)^{(-1)}$. Table 4.2 presents the absolute relative error for $M=4$ and $M=6$, using the present method at the same points as [3], together with the results obtained by wavelet Galerkin and wavelet collocation methods for $M=6$ given in [3]. 
Table 4.2. Absolute relative error for Example 4.2.

\begin{tabular}{ccccc}
\hline$x_{i}$ & $M=4$ & $M=6$ & wavelet Galerkin [3] & wavelet collocation [3] \\
\hline 0.000 & 0.0 & 0.0 & 0.0 & 0.0 \\
0.125 & $9.2 \times 10^{-6}$ & $2.5 \times 10^{-7}$ & $7.9 \times 10^{-7}$ & $9.4 \times 10^{-4}$ \\
0.250 & $4.2 \times 10^{-6}$ & $1.9 \times 10^{-7}$ & $1.3 \times 10^{-6}$ & $1.6 \times 10^{-3}$ \\
0.375 & $2.6 \times 10^{-5}$ & $1.4 \times 10^{-6}$ & $1.6 \times 10^{-6}$ & $2.0 \times 10^{-3}$ \\
0.500 & $3.9 \times 10^{-5}$ & $1.1 \times 10^{-6}$ & $1.7 \times 10^{-6}$ & $1.9 \times 10^{-3}$ \\
0.625 & $3.9 \times 10^{-5}$ & $4.7 \times 10^{-7}$ & $1.5 \times 10^{-6}$ & $1.7 \times 10^{-3}$ \\
0.750 & $2.9 \times 10^{-5}$ & $6.9 \times 10^{-7}$ & $1.2 \times 10^{-6}$ & $1.1 \times 10^{-3}$ \\
0.875 & $1.2 \times 10^{-5}$ & $2.1 \times 10^{-7}$ & $6.5 \times 10^{-7}$ & $5.5 \times 10^{-4}$ \\
1.000 & 0.0 & 0.0 & 0.0 & 0.0 \\
\hline
\end{tabular}

Table 4.3. Absolute relative error for Example 4.3.

\begin{tabular}{cccc}
\hline$x_{i}$ & $M=6$ & wavelet Galerkin [3] & wavelet collocation [3] \\
\hline 0.000 & 0.0 & 0.0 & 0.0 \\
0.125 & $1.8 \times 10^{-5}$ & $2.7 \times 10^{-4}$ & $2.6 \times 10^{-2}$ \\
0.250 & $1.5 \times 10^{-5}$ & $3.1 \times 10^{-5}$ & $1.6 \times 10^{-2}$ \\
0.375 & $1.2 \times 10^{-5}$ & $2.6 \times 10^{-4}$ & $9.3 \times 10^{-3}$ \\
0.500 & $1.0 \times 10^{-5}$ & $4.3 \times 10^{-4}$ & $5.2 \times 10^{-3}$ \\
0.625 & $7.9 \times 10^{-6}$ & $5.6 \times 10^{-4}$ & $2.5 \times 10^{-3}$ \\
0.750 & $6.0 \times 10^{-6}$ & $6.6 \times 10^{-4}$ & $1.0 \times 10^{-3}$ \\
0.875 & $3.7 \times 10^{-6}$ & $7.2 \times 10^{-4}$ & $2.4 \times 10^{-4}$ \\
0.100 & 0.0 & 0.0 & 0.0 \\
\hline
\end{tabular}

Example 4.3. Consider second-order Fredholm integro-differential equation [3]

$$
\begin{aligned}
& x^{2} y^{\prime \prime}(x)+50 x y^{\prime}-35 y(x) \\
& \quad=\frac{1-e^{(x+1)}}{x+1}+\left(x^{2}+50 x-35\right) e^{x}+\int_{0}^{1} e^{x t} y(t) d t, \quad y(0)=1, \quad y(1)=e .
\end{aligned}
$$

Exact solution of this problem is $e^{x}$. Table 4.3 presents the absolute relative error for $M=$ 6 , using the present method at the same points as [3], together with the results obtained by wavelet Galerkin and wavelet collocation methods for $M=6$ given in [3].

\section{Conclusion}

In the present work, a technique has been developed for solving nonlinear second-order Fredholm integro-differential equations. The method is based upon compactly supported linear semiorthogonal $B$-spline wavelets. The operational matrices of derivative for $B$ spline scaling functions and wavelets are given. The problem has been reduced to solving a system of nonlinear algebraic equations and applications are demonstrated through numerical examples. 


\section{Acknowledgment}

The work of the first author was supported by Iran Telecommunication Research Center.

\section{References}

[1] G. Ala, M. L. Di Silvestre, E. Francomano, and A. Tortorici, An advanced numerical model in solving thin-wire integral equations by using semi-orthogonal compactly supported spline wavelets, IEEE Transactions on Electromagnetic Compatibility 45 (2003), no. 2, 218-228.

[2] A. Ayad, Spline approximation for first order Fredholm integro-differential equations, Universitatis Babeș-Bolyai. Studia. Mathematica 41 (1996), no. 3, 1-8.

[3] S. H. Behiry and H. Hashish, Wavelet methods for the numerical solution of Fredholm integrodifferential equations, International Journal of Applied Mathematics 11 (2002), no. 1, 27-35.

[4] C. K. Chui, An Introduction to Wavelets, Wavelet Analysis and Its Applications, vol. 1, Academic Press, Massachusetts, 1992.

[5] J. C. Goswami, A. K. Chan, and C. K. Chui, On solving first-kind integral equations using wavelets on a bounded interval, IEEE Transactions on Antennas and Propagation 43 (1995), no. 6, 614622.

[6] M. Lakestani, M. Razzaghi, and M. Dehghan, Solution of nonlinear Fredholm-Hammerstein integral equations by using semiorthogonal spline wavelets, Mathematical Problems in Engineering 2005 (2005), no. 1, 113-121.

[7] P. Linz, A method for the approximate solution of linear integro-differential equations, SIAM Journal on Numerical Analysis 11 (1974), no. 1, 137-144.

[8] G. Micula and G. Fairweather, Direct numerical spline methods for first-order Fredholm integrodifferential equations, Revue d'Analyse Numérique et de Théorie de l'Approximation 22 (1993), no. 1, 59-66.

[9] R. D. Nevels, J. C. Goswami, and H. Tehrani, Semi-orthogonal versus orthogonal wavelet basis sets for solving integral equations, IEEE Transactions on Antennas and Propagation 45 (1997), no. 9, 1332-1339.

[10] G. M. Phillips, Analysis of numerical iterative methods for solving integral and integrodifferential equations, The Computer Journal 13 (1970), no. 3, 297-300.

[11] A. Ralston and P. Rabinowitz, A First Course in Numerical Analysis, McGraw-Hill, New York, 1985.

[12] W. Volk, The numerical solution of linear integro-differential equations by projection methods, Journal of Integral Equations 9 (1985), no. 1, suppl., 171-190.

M. Lakestani: Department of Applied Mathematics, Amirkabir University of Technology,

Tehran 15914, Iran

E-mail address: lakestani@aut.ac.ir

M. Razzaghi: Department of Mathematics and Statistics, Mississippi State University, MS 39762, USA Current address: Department of Applied Mathematics, Amirkabir University of Technology,

Tehran 15914, Iran

E-mail address: razzaghi@math.msstate.edu

M. Dehghan: Department of Applied Mathematics, Amirkabir University of Technology,

Tehran 15914, Iran

E-mail address: mdehghan@cic.aut.ac.ir 


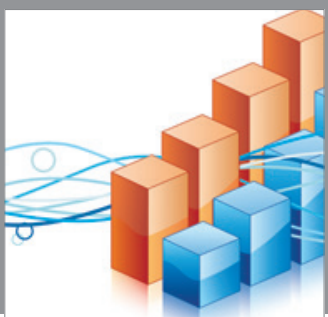

Advances in

Operations Research

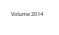

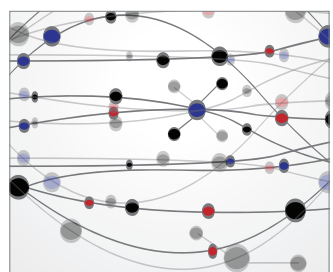

\section{The Scientific} World Journal
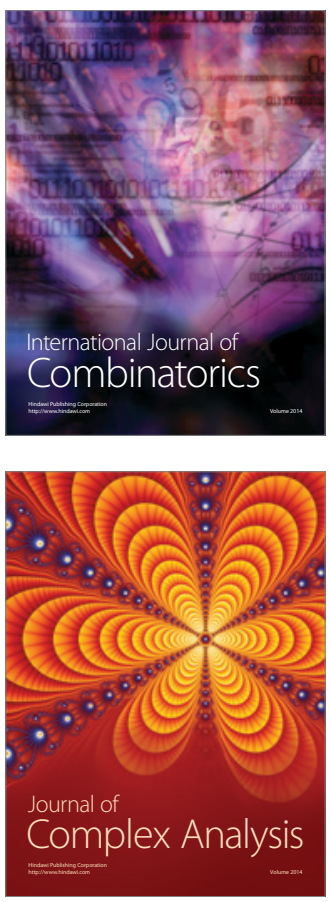

International Journal of

Mathematics and

Mathematical

Sciences
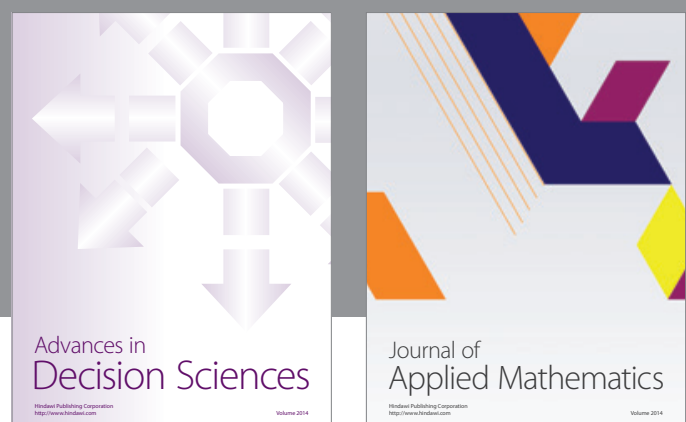

Journal of

Applied Mathematics
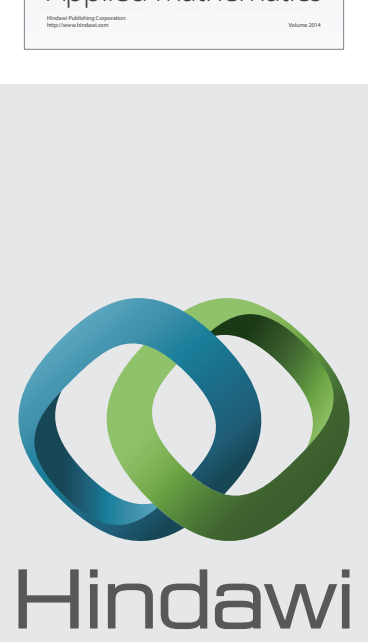

Submit your manuscripts at http://www.hindawi.com
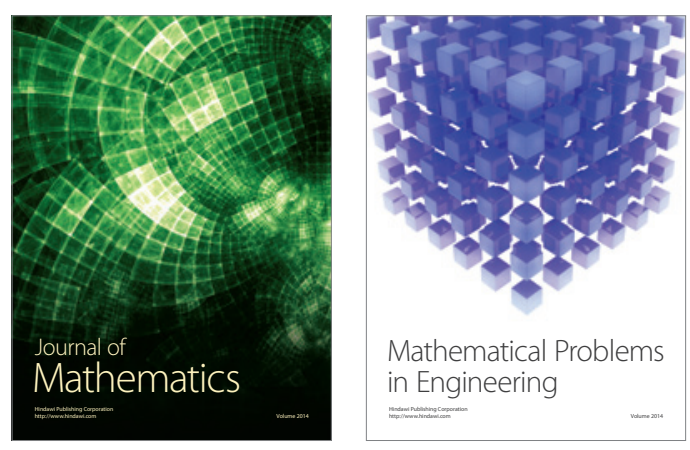

Mathematical Problems in Engineering
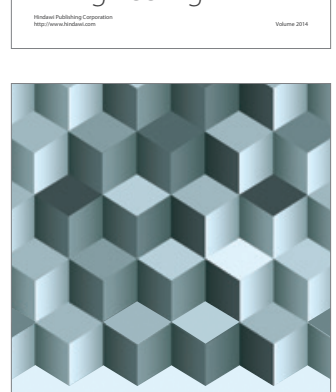

Journal of

Function Spaces
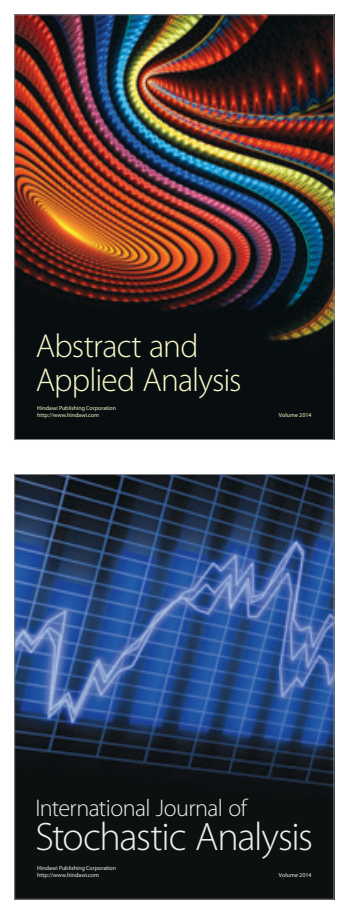

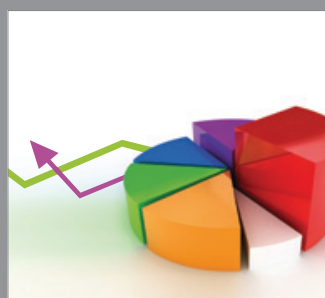

ournal of

Probability and Statistics

Promensencen
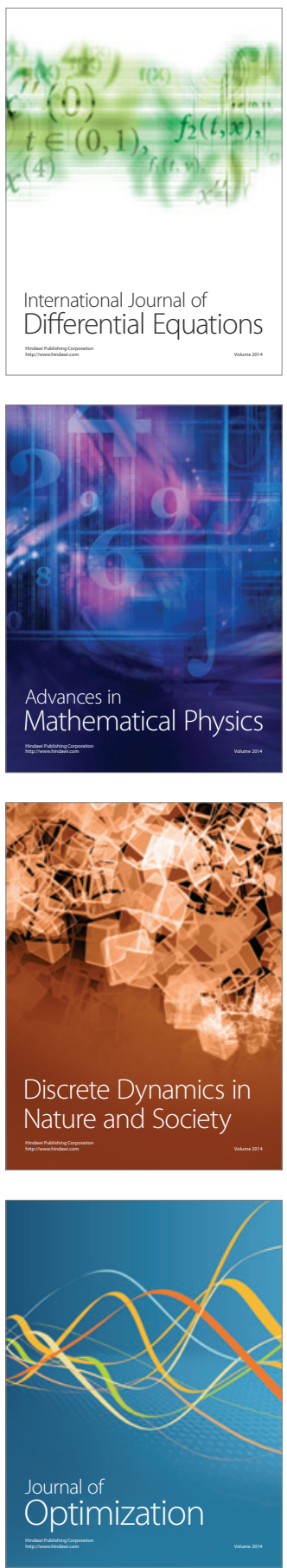